\title{
Recurrent Respiratory Tract Infection in an infant: Early Diagnosis by Clinical and Radiological Pearls
}

\section{Shatanik Sarkar ${ }^{1}$, Chaitali Patra ${ }^{2}$, Shibani Pal ${ }^{1}$, and Arkapriya Pramanik}

${ }^{1}$ Department of Paediatric Medicine, R. G. Kar Medical College and Hospital, Kolkata, India

${ }^{2}$ Department of Paediatric Medicine, Tapan Sinha Memorial Hospital, Kolkata, India

${ }^{3}$ Department of Paediatric Medicine, Malda Medical College and Hospital, Malda

\section{Correspondence:}

Shatanik Sarkar

Department of Paediatric Medicine,

R. G. Kar Medical College and Hospital,

Deshbandhunagar, Baguiati, Kolkata - 59 .

India

E-mail id: baselinegame@gmail.com

DOI: $10.3126 /$ jnps.v41i1.30250

Submitted on: 2020-06-15

Accepted on: 2020-10-04

Acknowledgements: None

Funding: Nil

Conflict of Interest: None declared

Permission from IRB: Yes
To cite this article: Sarkar S, Patra C, Pal S, Pramanik A. Recurrent Respiratory Tract Infection in an infant: Early Diagnosis by Clinical and Radiological Pearls. J Nepal Paediatr Soc. 2021;41(1):111-4.

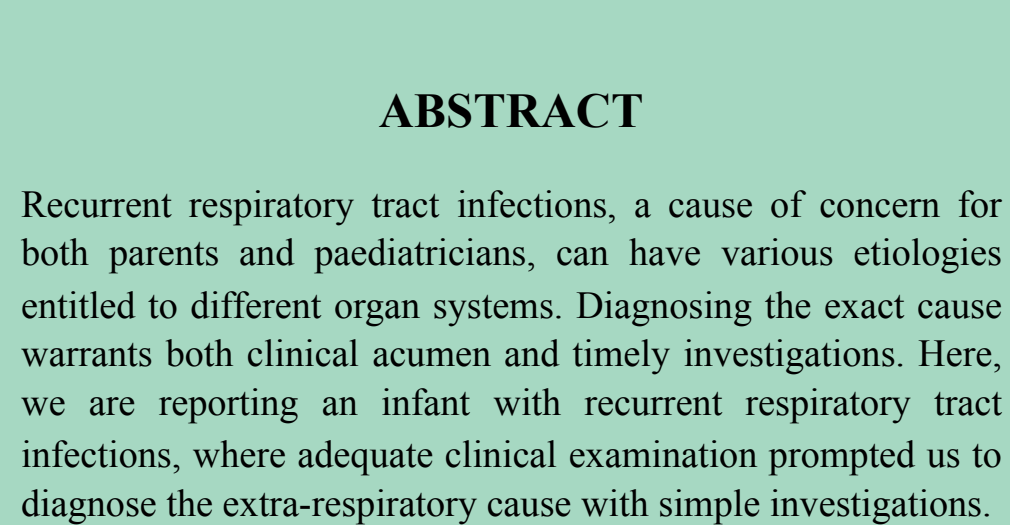

Keywords: Joubert Syndrome; Molar tooth sign; Recurrent RTI

This work is licensed under creative common attribution 3.0 license 


\section{INTRODUCTION}

Recurrent respiratory tract infections (RTI) are one of the leading reasons for OPD consultation and hospitalisation in children, particularly the infants. More than six serious respiratory tract diseases a year are termed as recurrent RTI (RRI). ${ }^{1}$ Epidemiologists estimate that approximately $15 \%$ of children suffer from RRI. ${ }^{1}$ Though the disease mainly involves upper respiratory tract, involvement of lower respiratory tract (i.e. LRTI or pneumonia) is more serious and more often requires hospitalisation. Apart from diseases of the respiratory tract (e.g. congenital malformations, defective mucociliary clearance etc.), causes of RRI may also include cardiovascular diseases, aspiration, immunodeficiencies and neurological ailments. Here, we are presenting such a case of recurrent RTI in an infant, where the cause was lying elsewhere.

\section{CASE REPORT}

A 10 month old female child presented to our hospital OPD with complaints of repeated chest infections since birth. She had been hospitalised five times for breathlessness with or without fever since the age of one month. She was born at term out of non-consanguineous marriage with uneventful prenatal and natal periods. However, mother complained of feeding difficulties and occasional fast breathing from the immediate postnatal period. No convulsion was ever seen. There was obvious delay in achieving the motor milestones, though language and social milestones were almost normal. There was no history of similar illness in any of the two elder siblings.

On examination, her weight was below $3^{\text {rd }}$ percentile, but her length and head circumference were within normal limits. Facies of the child was insignificant, except the presence of prominent forehead. Respiratory system examination was essentially normal with presence of bilateral vesicular breath sounds without any adventitious sounds. But while examining the child for prolonged period, breathing pattern seemed abnormal with brief episodes of hyperpnoea interspersed with normal pattern. Neurologically, the child had generalised hypotonia, but deep tendon reflexes were normal and plantars were equivocal. Other systems including cardiovascular system didn't reveal any significant abnormalities. Ocular and ophthalmoscopic examinations were also normal.

Routine investigations including $\mathrm{CBC}$ and chest $\mathrm{X}$ ray (CXR) were within normal limits. All the previous CXRs available with the parents also didn't show any feature of pneumonia. Keeping in mind the abnormal neurological findings on clinical examination, MRI of brain was ordered. Axial T1weighted image showed the features of hypoplastic cerebellar vermis with elongated and thick superior cerebellar peduncles giving it the appearance of "molar tooth" in midbrain (Molar Tooth Sign or MTS) [Figure 1A]. T2-weighted image showed "bat-wing" shaped $4^{\text {th }}$ ventricle due to dilatation of the same [Figure 1B]. Echocardiography and abdominal ultrasonography were normal. Based on the clinical features and neuroimaging, a diagnosis of Joubert Syndrome (JS) was made.

\section{DISCUSSION}

JS is a rare congenital CNS malformation with autosomal recessive inheritance in most of them, though $\mathrm{X}$-linked recessive cases has been reported. ${ }^{2,3}$ It was first described by French neurologist Marie Joubert in 1969 in four siblings with agenesis of cerebellar vermis presenting with episodic hyperpnea, abnormal eye movements, ataxia and intellectual disability. ${ }^{4}$ Several years later, the pathognomic midbrain-hindbrain malformation giving rise to the MTS in radiography was detected in JS.

Even in the absence of established diagnostic criteria, hypotonia, developmental delay, and one or both of the following - a) Irregular breathing pattern, b) Abnormal eye movements have been mentioned as essential for diagnosis of JS along with MRI signs. ${ }^{2}$ MRI plays a cornerstone in establishing the diagnosis of JS, because presence of MTS in cranial imaging is very much specific of this disease. Presence of abnormally deep interpeduncular fossa, elongated thick and maloriented superior cerebellar peduncles and absence or hypoplasia of cerebellar vermis gives the characteristic appearance of MTS. ${ }^{5}$ Subsequently, the fourth ventricle is moderately 


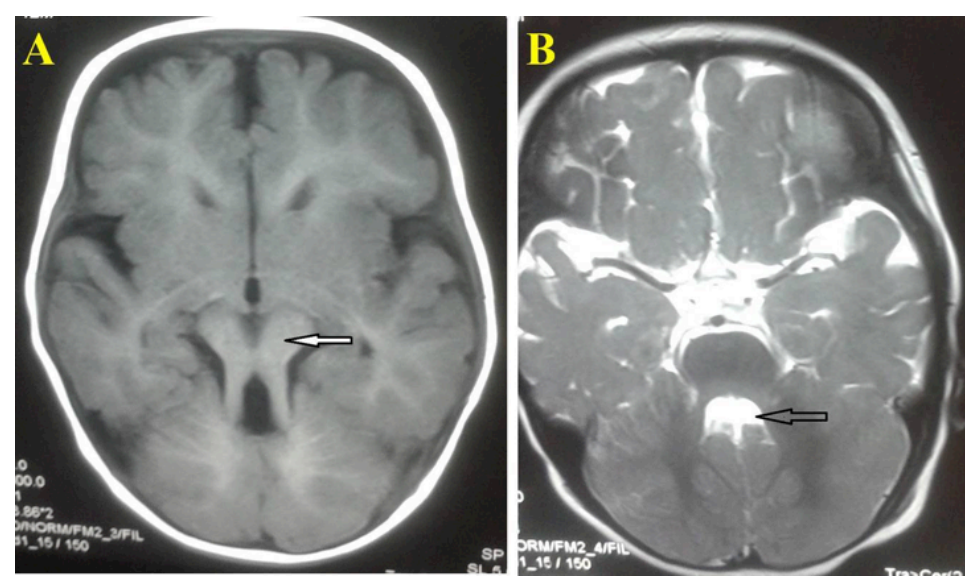

Figure 1. [A] Axial T1-weighted MRI of brain showing features of hypo plastic cerebellar vermis with thickening of superior cerebellar peduncles giving it the appearance of "molar tooth" in midbrain.

[B] T2 weighted image in MRI of brain showing 'batwing' shaped 4th ventricle.

dilated taking the appearance of a "bat wing" or an open "umbrella" on axial CT/MRI. These two signs are reported to be consistent and reliable imaging findings to distinguish JS from other posterior fossa abnormalities. The possibility of prenatal diagnosis of JS has been reported with the ultrasonographic findings showing increased nuchal translucency. ${ }^{6}$

Joubert Syndrome Related Disorders (JSRD) is a group of disorders with developmental delay and multiple congenital anomalies involving mainly retina, kidney, liver and skeleton. The marked pleiotropism of JSRD can be explained by the 10 causative genes identified till date - JBTS1 to JBTS10. They all encode for proteins of primary cilium making JSRD part of an expanding group of diseases called "Ciliopathies."7 These proteins play key role in the development and functioning of retinal photoreceptors, neurons, kidney tubules and bile ducts. ${ }^{8}$ In the developing cerebellum and brainstem these organelles regulating major signal transduction pathways have been implicated both in neuronal cell proliferation and axonal migration. ${ }^{9}$

Clinically, JSRDs are classified into six phenotypic subgroups: Pure JS, JS with ocular defect, JS with renal defect, JS with oculorenal defect, JS with hepatic defect and JS with orofaciodigital defect. ${ }^{2}$ Thus, diagnosis of JS by MTS in radiography should be followed by a diagnostic protocol to assess whether there is any other organ involvement. Prognosis also largely depends on the severity of the involvement of different organ systems, particularly retina, liver and kidney. There was no extraneuronal involvement in our case suggesting it as a case of Pure JS.

Recurrent RTI, as seen in our case, can be attributed to various factors, e.g. feeding difficulty causing micro-aspirations, hypotonia of respiratory muscles denying adequate cough response, or the ciliary abnormality also affecting the respiratory cilia. The breathing pattern in JS, an effortless hyperventilation in episodes, may have also contributed to overestimation of respiratory distress causing multiple hospitalisations in this case.

\section{CONCLUSIONS}

Considering the various etiologies of recurrent RTI involving multiple systems other than the respiratory system, careful clinical evaluation is of paramount importance before jumping into the battery of tests. Though there is no curative therapy for JS, early diagnosis dictates prognostic outcome and an opportunity for genetic counselling. 


\section{REFERENCES}

1. Raniszewska A, Gorska E, Kotula I, Stelmaszczyk-Emmel A, Popko K, Ciepiela O. Recurrent respiratory tract infections in children - analysis of immunological examinations. Cent Eur J Immunol. 2015;40(2):167-73. DOI: $10.5114 /$ ceji.2015.52830

2. Singh P, Goraya JS, Saggar K, Ahluwalia A. A report of Joubert Syndrome in an infant, with literature review. J Pediatr Neurosci. 2011;6:44-7. DOI: 10.4103/1817-1745.84407

3. Coene KL, Roepman R, Doherty D, Afroze B, Kroes HY, Letteboer SJF, et al. OFD1 is mutated in X-linked Joubert syndrome and interacts with LCA5-encoded lebercilin. Am J Hum Genet. 2009;85(6):465-81. DOI: 10.1016/j.ajhg. 2009.09.002

4. Joubert M, Eisenring JJ, Robb JP, Andermann F. Familial agenesis of the cerebellar vermis. A syndrome of episodic hyperpnea, abnormal eye movements, ataxia, and retardation. Neurology. 1969;19:813-25. DOI: 10.1212/wnl. 19.9.813

5. Romani M, Micalizzi A, Valente EM. Joubert syndrome: congenital cerebellar ataxia with the molar tooth. Lancet Neurol. 2013;12(9):894-905. DOI: 10.1016/S1474-4422(13)70136-4

6. Aslan H, Ulker V, Gulcan EM, Numanoglu C, Gul A, Agar M, et al. Prenatal diagnosis of Joubert syndrome: A case report. Prenat Diagn. 2002;22:13-6. DOI: 10.1002/pd.220

7. Brancati F, Dallapiccola B, Valente EM. Joubert syndrome and related disorders. Orphanet J Rare Dis. 2010;5:20. DOI: $10.1186 / 1750-1172-5-20$

8. Badano JL, Mitsuma N, Beales PL, Katsanis N. The Ciliopathies: An Emerging Class of Human Genetic Disorders. Annu Rev Genomics Hum Genet. 2006;7:125-48. DOI: 10.1146/annurev.genom.7.080505.115610

9. Millen KJ, Gleeson JG. Cerebellar development and disease. Curr Opin Neurobiol. 2008;18:12-9. DOI: 10.1016/ j.conb.2008.05.010 